\title{
Investigaciones sobre sostenibilidad de los recursos naturales en el Instituto Nacional de Investigaciones Forestales, Agrícolas y Pecuarias
}

\section{Research on the sustainability of natural resources at the Instituto Nacional de Investigaciones Forestales, Agrícolas y Pecuarias}

\author{
Marisela Cristina Zamora-Martínez ${ }^{1}$, Aixchel Maya-Martínez ${ }^{2 *}$, Nelda Guadalupe \\ Uzcanga-Pérez ${ }^{3}$, Rosario Rogel-Salazar ${ }^{4}$, Irvin Santiago-Bautista ${ }^{5}$, Guadalupe \\ Fabiola Reygadas Prado ${ }^{6}$ y Eulalia Edith Villavicencio-Gutiérrez ${ }^{7}$
}

\begin{abstract}
This paper summarizes the available knowledge generated at the Instituto Nacional de Investigaciones Forestales, Agrícolas $y$ Pecuarias (INIFAP) on the sustainability of natural resources. A documentary search was performed in the Scopus (1972-2020) and Web of Science (WoS) databases, as well as in the SciELO Citation Index sub-database (2002-2020) and the Mexican Journal of Forestry (Revista Mexicana de Ciencias Forestales, RMCF) (1985-2009). The information was drawn based on 10 keywords and was analyzed using the Bibliometrix, VOSviewer and IBM-SPSS 23.0 software to identify the formation of collaboration networks and co-authorships. The publications generated by forestry researchers on sustainability issues are incipient, and most are from the present century; 3653 documents were initially recovered from Scopus, and 210 in a second instance; 1447 in WoS, and 28 in $R M C F$. The above coincides with the rise of global interest in climate change and its impact on ecosystems and biodiversity, as well as in the importance of ecosystem services and sustainable forest management. Other relevant aspects are water management in agroecosystems and integrated basin management. Five collaborative networks were identified in Scopus, 15 in WoS, and 4 in the RMCF. INIFAP has human resources with experience and knowledge in the three sectors it comprises, as well as specialists in socio-economics; this constitutes a strength for the formation of multisectoral collaborations to undertake visionary projects concerning the sustainable development of natural resources.
\end{abstract}

Key words: Climate change, scientometrics, basin management, collaborative networks, sustainable development, sustainability.

\section{Resumen}

Se presenta una síntesis del conocimiento generado en el Instituto Nacional de Investigaciones Forestales, Agrícolas y Pecuarias (INIFAP) sobre sostenibilidad de los recursos naturales, a partir de una investigación documental en las bases de datos Scopus (1972-2020), Web of Science (WoS), en la sub base SciELO Citation Index (2002-2020) y la Revista Mexicana de Ciencias Forestales (RMCF) (1985-2009). Se utilizaron 10 palabras clave, y el análisis se hizo con los programas Bibliometrix, VOSviewer e IBM-SPSS 23.0 para la integración de redes de colaboración e identificación de coautorías. Destaca que las publicaciones de los investigadores forestales son escasas en las bases consultadas y la mayoría data del presente siglo; se recuperaron, inicialmente, 3653 documentos en Scopus, y en una segunda instancia 210; en WoS, 1 447; y en RMCF, 28. Lo anterior coincide con el despunte del interés global por el cambio climático, su impacto en los ecosistemas y la biodiversidad; así como la importancia de los servicios ecosistémicos y el manejo forestal sustentable. Otro aspecto relevante es el manejo del agua en los agroecosistemas y el manejo integrado de cuencas. Se identificaron cinco redes de colaboración en Scopus, 15 en WoS y cuatro en la RMCF. El INIFAP tiene un capital humano con experiencia y conocimientos en los tres sectores que lo integran; así como de especialistas en socioeconomía, que constituyen una fortaleza para la conformación de colaboraciones multisectoriales para la realización de proyectos de gran visión sobre el desarrollo sostenible de los recursos naturales.

Palabras clave: Cambio Climático, cienciometría, manejo de cuencas, redes de colaboración, sostenibilidad, sustentabilidad. Fecha de recepción/Reception date: 5 de marzo de 2021

Fecha de aceptación/Acceptance date: 11 de junio de 2021

\footnotetext{
${ }^{1}$ Instituto Nacional de Investigaciones Forestales, Agrícolas y Pecuarias. Centro Nacional de Investigación Disciplinaria en Conservación y Mejoramiento de Ecosistemas Forestales. México.

${ }^{2}$ Instituto Nacional de Investigaciones Forestales, Agrícolas y Pecuarias. CIR-Sureste, Campo Experimental Edzná. México.

${ }^{3}$ Instituto Nacional de Investigaciones Forestales, Agrícolas y Pecuarias. CIR-Sureste, Campo Experimental Mocochá. México.

${ }^{4}$ Universidad Autónoma del Estado de México. Facultad de Ciencias Políticas y Sociales. México.

5Universidad Autónoma del Estado de México. México.

${ }^{6}$ Instituto Nacional de Investigaciones Forestales, Agrícolas y Pecuarias. CIR- Pacífico Centro, Campo Experimental Centro Altos de Jalisco. México

${ }^{7}$ Instituto Nacional de Investigaciones Forestales, Agrícolas y Pecuarias. CIR-Noreste, Campo Experimental Saltillo. México.

*Autor para correspondencia; correo-e: maya.aixchel@inifap.gob.mx
} 


\section{Introducción}

La especie humana desde su origen ha dependido para su desarrollo y evolución cultural de la transformación de los ecosistemas y de los diversos servicios que estos le proporcionan (CONABIO, 2008). En este contexto, a nivel mundial, la protección y conservación de los ecosistemas es, actualmente, de máxima prioridad debido a las presiones demográficas que conllevan a un alto requerimiento de los servicios ecosistémicos, así como por los cambios de uso de la tierra para satisfacer una creciente demanda de alimentos (Arnold et al., 2011).

Asimismo, es importante considerar que el desarrollo económico de la sociedad global tiene su origen en tecnologías que, generalmente, resultan contaminantes y adversas para la conservación del ambiente. Esto ha originado la degradación de las áreas forestales y sus servicios ecosistémicos, con la consecuente exacerbación de la pobreza de los habitantes de esas zonas; hechos que, a la luz del conocimiento actual, tienden a persistir y, aún más, a empeorar (Chopra et al., 2005).

En este contexto, surge el concepto de sostenibilidad del Informe Brundtland (ONU, 1987): ..." El desarrollo que satisface las necesidades del presente sin comprometer la posibilidad de que las futuras generaciones satisfagan sus propias necesidades...". En los mismos términos, la Conferencia de las Naciones Unidas sobre el Medio Ambiente (FAO, 1992) acordó que los conceptos generales del manejo forestal sostenible son un elemento importante para lograr el desarrollo: "...Los recursos forestales y los terrenos forestales se deberían manejar de manera sostenible para satisfacer las necesidades sociales, económicas, ecológicas, culturales y espirituales de la presente generación y de las futuras generaciones...".

Es necesario señalar que a partir del Informe de Brundtland se hizo la traducción del término sustainable development como desarrollo sostenible, lo cual generó la confusión si hacen referencia a lo mismo o no los términos "desarrollo sostenible" y "desarrollo sustentable". La única diferencia es el origen de la traducción al español: 
en el caso de México se tradujo como desarrollo sostenible y en otros países de habla hispana, como desarrollo sustentable (Zarta, 2018).

El concepto de sustentabilidad se introdujo en 1990 para simbolizar gráficamente la relación entre los objetivos: crecimiento económico, equidad social y sustentabilidad ambiental. Esto dio lugar al denominado Triángulo de Nijkamp, cuya área central es el desarrollo sustentable, que se logra, hipotéticamente, cuando los tres objetivos antes citados se alcanzan de manera simultánea (Zarta, 2018).

Sin embargo, desde la década de los 80 , cuando se acuñó el concepto de desarrollo sostenible (ONU, 1987), y hasta la fecha, su diferenciación con respecto a la sustentabilidad no es clara; incluso los investigadores los utilizan de manera indistinta. Salas-Zapata et al. (2017) señalan que en alrededor de $91 \%$ de los artículos revisados no se definió el concepto de sustentabilidad y en casi $60 \%$, se usaron de manera indiferenciada sustentabilidad y desarrollo sostenible.

La diferencia entre ambos conceptos se marca al definir que la sustentabilidad es una condición dinámica de un ecosistema que refleja las formas en que sus componentes bióticos y abióticos se interrelacionan; lo cual se vincula con la naturaleza homeostática de los sistemas socioecológicos (Barton y Gutiérrez-Antinopai, 2020). Mientras que, el desarrollo sustentable es una forma de producción que se basa en el potencial tanto de la naturaleza, como de la cultura y no en las leyes teóricas y pragmáticas del mercado; lo anterior implica que la sustentabilidad integra las perspectivas ambientales, económicas, éticas, gobernanza sustentable, institucionales y culturales (Viso, 2005; Waas et al., 2011).

En conclusión, el desarrollo sostenible pretende armonizar el proceso económico con la conservación de la naturaleza que, además, favorece un balance entre la satisfacción de necesidades actuales y las de las generaciones futuras (RiveraHernández et al., 2017), mediante el crecimiento económico sostenible sobre la naturaleza limitada de los recursos naturales (Barrios et al., 2007). En cambio, el desarrollo sustentable integra el potencial ecológico, el desarrollo tecnológico, la 
cultura y la sociedad para satisfacer las necesidades básicas de esta y mejorar su calidad de vida (López et al., 2015; Rivera-Hernández et al., 2017).

En ese contexto, en el Programa de Desarrollo del Instituto Nacional de Investigaciones Forestales, Agrícolas y Pecuarias (INIFAP, 2018) se consignan como retos para conseguir la conservación de los ecosistemas y la sostenibilidad de los recursos naturales, entendidos como los de tipo renovable (agua, clima, suelo y biodiversidad), el aprovechamiento racional del agua, tanto de temporal como de riego, en las actividades agropecuarias y forestales; así como la variabilidad del clima y sus efectos, positivos y negativos, en la fisiología de las plantas; el aumento de la presencia y frecuencia de plagas y enfermedades; la desertificación, pérdida de la fertilidad de los suelos; el incremento en la incidencia y frecuencia de incendios forestales; además del manejo de los recursos maderables, no maderables y de la fauna.

En el escenario antes planteado y bajo la premisa de que es importante sintetizar el conocimiento disponible para tener una opinión fundamentada del estado de los logros y limitaciones en lo que se refiere a la sostenibilidad de los recursos naturales, con la finalidad de identificar los temas en los que se requiere generar información de base científica que contribuya a un desarrollo compatible con el bienestar social y el manejo de los recursos naturales, en el presente trabajo se identifican aspectos relevantes de la investigación científica en el INIFAP sobre la sostenibilidad de los recursos naturales, y se muestra la evidencia disponible sobre el conocimiento generado en el Instituto desde su creación, en 1985, hasta enero de 2021. La información se presenta diferenciada por revistas e instituciones, artículos y redes de colaboración científica.

\section{Criterios de búsqueda}

La revisión temática comprendió el período de 1985 a 2020, excepto cuando se indique lo contrario, esto en el marco del XXXV aniversario del INIFAP. Se realizó una consulta sobre los recursos: suelo, agua, biodiversidad, servicios ambientales y clima, en las bases de datos: Scopus (https://www.scopus.com), Web of Science (WoS), 
SciELO Citation Index, (https://clarivate.com/webofsciencegroup/solitions/webofsciencescielo) y la Revista Mexicana de Ciencias Forestales (https://cienciasforestales.inifap.gob.mx) (RMCF). Las etapas del proceso de revisión bibliográfica se describen en la Figura 1.

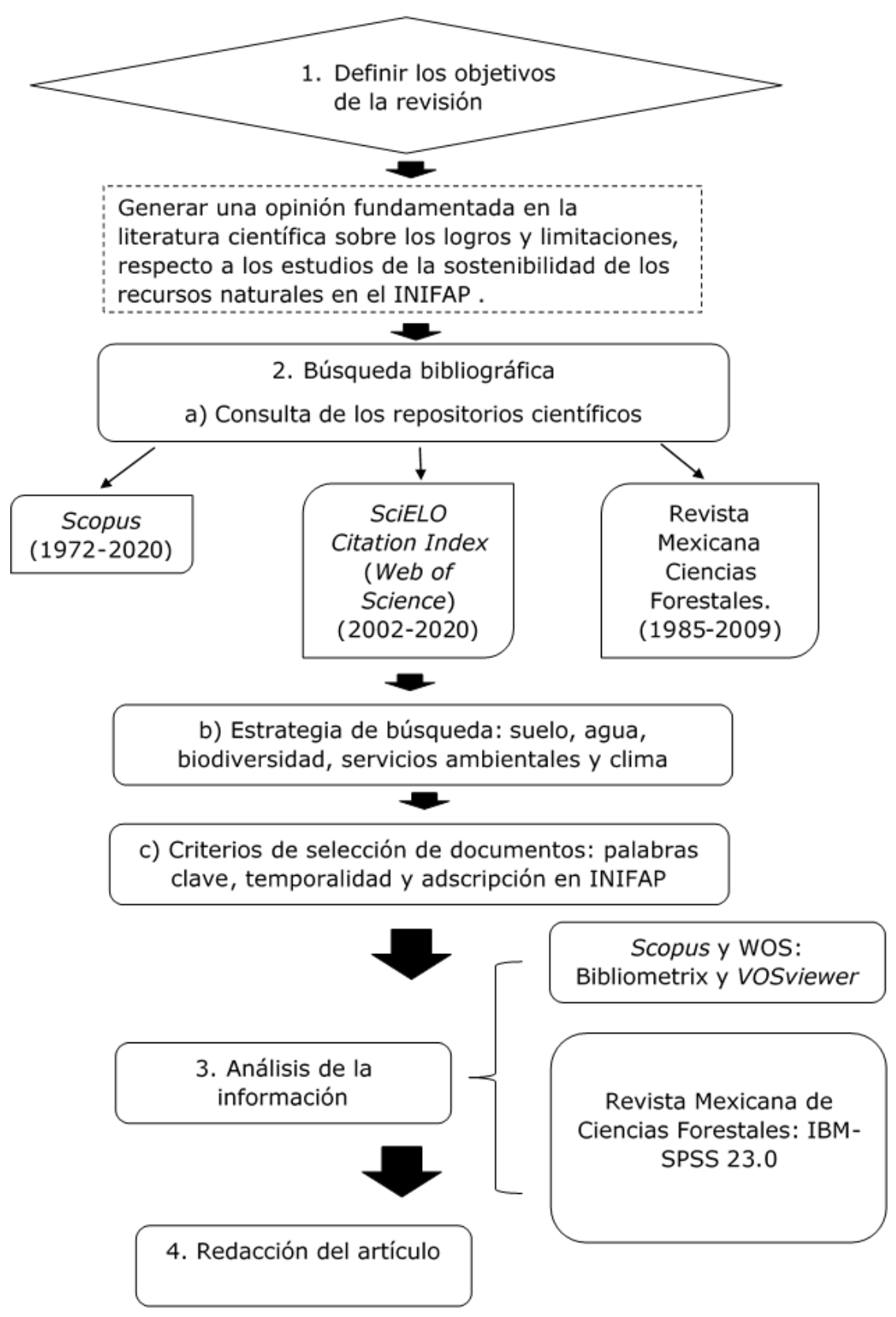

Figura 1. Esquema de trabajo. 
En primera instancia, se construyó un operador de búsqueda en torno a los temas de Sostenibilidad de los Recursos Naturales considerados en el Programa de Desarrollo del Instituto Nacional de Investigaciones Forestales, Agrícolas y Pecuarias (INIFAP, 2018) con diez palabras clave: Basin River, Biodiversity Management, Forest Soil Conservation, Sustainable Forest Management, Climate Change, Forest Restoration, Integrated Pest Management, Fire Management, Forest Plantations, y Ecosystem Conservation. Posteriormente, se amplió la búsqueda con la integración de términos más específicos: Basin Water, Biodiversity, Soil, Sustainable, Climate Change, Restoration, Integrated Pest Management, Fire Management, Forest Plantations y Ecosystem Conservation. Además, el criterio de selección para la búsqueda en las bases de datos fue considerar al menos un autor que declarara estar adscrito al INIFAP, o haberlo estado durante el periodo de estudio.

Para el criterio temporal, se consideró la cobertura total de las bases de datos Scopus y Scielo Citation Index. Para el caso de Scopus, la cobertura comprende de 1972 hasta 2020, mientras que el de Scielo Citation Index contempla sólo de 2002 a 2020, período para el cual WoS integra información, ya que para 2021 aún no se habían agregado datos, al momento de la consulta. Para la cobertura de la RMCF, se revisó de 1985 a 2009, ya que a partir de 2010 la Revista se incorporó a Scielo Citation Index.

Los registros obtenidos de Scopus y WoS se analizaron con los paquetes estadísticos: Bibliometrix (Aria y Cuccurullo, 2017) y VOSviewer (Van Eck y Waltman, 2007) para calcular porcentajes, redes de ocurrencia de palabras clave, tendencias y dinámicas de crecimiento, así como las redes de coautorías.

Para la información procedente del portal de la RMCF se usó el programa informático Statistical Package for the Social Sciencies (IBM-SPSS) 23.0 (IBM, 2015), con el cual se estimaron las frecuencias por año de publicación, por programa de investigación del INIFAP y tipo de recurso natural al que están contribuyendo para su sostenibilidad. 


\section{Análisis de la información}

Una búsqueda inicial en los registros de Scopus sobre publicaciones con autoría de investigadores del INIFAP y sus institutos precedentes: Instituto Nacional de Investigaciones Forestales (INIF), Instituto Nacional de Investigaciones Agrícolas (INIA) e Instituto Nacional de Investigaciones Pecuarias (INIP), de 1972 hasta 2020, produjo un total de 3653 documentos; cuando esta búsqueda se restringió al primer grupo de palabras clave se obtuvieron 32 documentos. Posteriormente, al ampliarla con las nuevas palabras clave, se recuperaron 210 documentos publicados entre los años 2000 y enero de 2021 . Es decir, en todo el acervo histórico de la revista Scopus antes del año 2000, no hay mención de los temas buscados en palabras clave, títulos y resúmenes.

En el caso de WoS, para eliminar el sesgo de la subrepresentación de la región Iberoamericana en la Colección Principal, se realizó la búsqueda en la sub-base de datos Scielo Citation Index, que se alimenta directamente de las Colecciones de SciELO en Iberoamérica. Para el período 2002-2020 mediante este análisis se localizaron 1447 publicaciones firmadas por al menos un autor cuya adscripción institucional fue el INIFAP.

Para el período 1985-2009 de la RMCF, se obtuvieron 28 artículos relacionados con la Sostenibilidad de los Recursos Naturales.

\section{Revisión documental de la base de datos Scopus (1972 a 2020)}

El análisis de la información recabada en la base total de Scopus evidenció que los temas centrales de las publicaciones de los investigadores adscritos al INIFAP y sus institutos precedentes: INIF, INIA e INIP, se relacionaron con las palabras clave: maíz, rendimiento y ganado (Figura 2). 


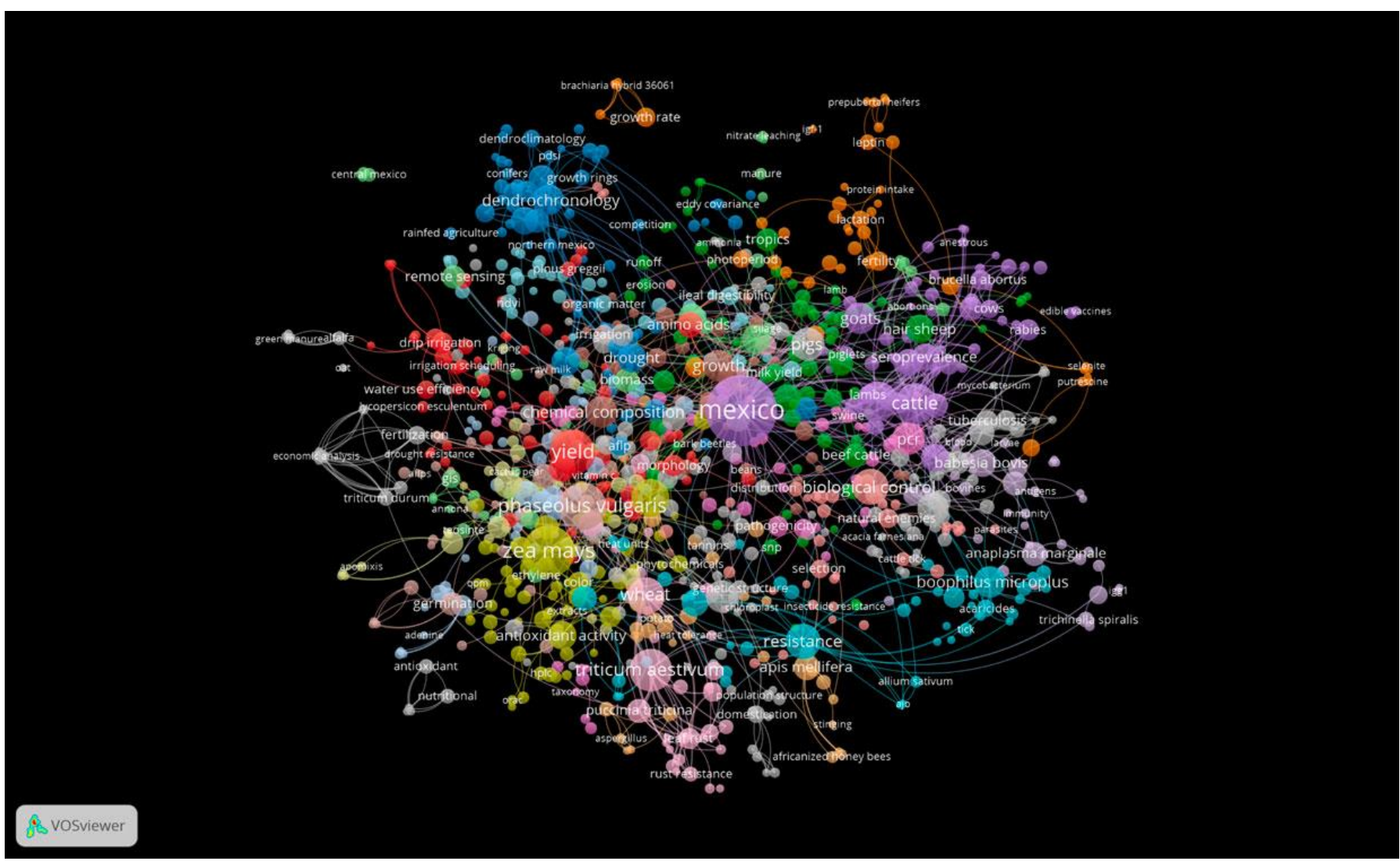

Fuente: elaboración propia con base en Scopus (1972-2020) y creado con VOSviewer.

Figura 2. Red de coocurrencia de la producción total indizada en Scopus de investigadores adscritos a los institutos INIF, INIA, INIP e INIFAP (1972-2020).

Entre los temas más visibles no se localizó ninguno relacionado con estudios de Sostenibilidad de los Recursos Naturales; sin embargo, esto no quiere decir que haya estado fuera de la atención de los investigadores, ya que en los 3653 documentos compilados se advierten tópicos de alguna manera relacionados con estudios referentes a esa temática; entre ellos, los más significativos fueron: cambio climático, sequía y diversidad genética, con $2 \%$ cada uno; manejo forestal, diversidad y variabilidad climática (1\%).

En la Figura 3 se observa cómo, a partir de la segunda década del siglo XXI, los temas relacionados con estudios de Sostenibilidad de los Recursos Naturales empiezan a despuntar. Por ejemplo, entre los tópicos tendencia en la producción editorial del INIFAP destacaron cambio climático, el cual sobresale a partir de 2019. Además, 
desde 2018 se aprecia una dinámica de crecimiento de las palabras clave: manejo forestal, diversidad genética, inventario forestal, entre otras, a diferencia de las palabras clave asociadas a estudios de índole agrícola o pecuaria, las cuales prevalecieron entre 2012 y 2016.

\section{Trend Topics}

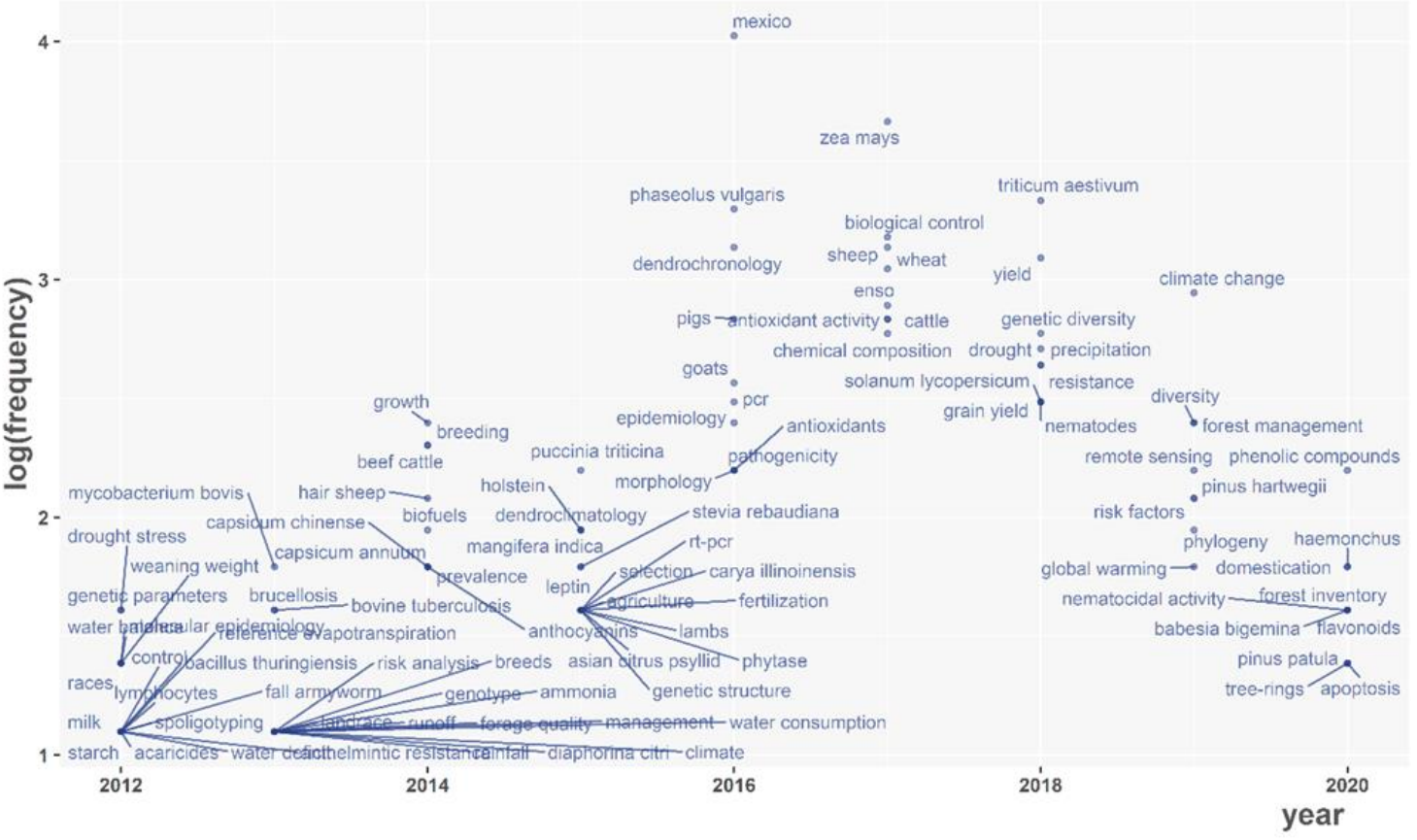

Fuente. Elaboración propia con base en Scopus (2020), creado con Bibliometrix.

Figura 3. Tópicos tendencia en la producción total de investigadores adscritos a los institutos INIF, INIA, INIP e INIFAP indizada en Scopus (1972-2021).

Con base en los 210 artículos localizados en la base de datos Scopus entre 2000 y 2021, se construyó el mapa temático que se muestra en la Figura 4, donde se aprecia que el nodo temático central fue México, seguido en orden de importancia por cambio climático y sequía; también, se identificaron manejo forestal, productividad y su relación con el agua. 


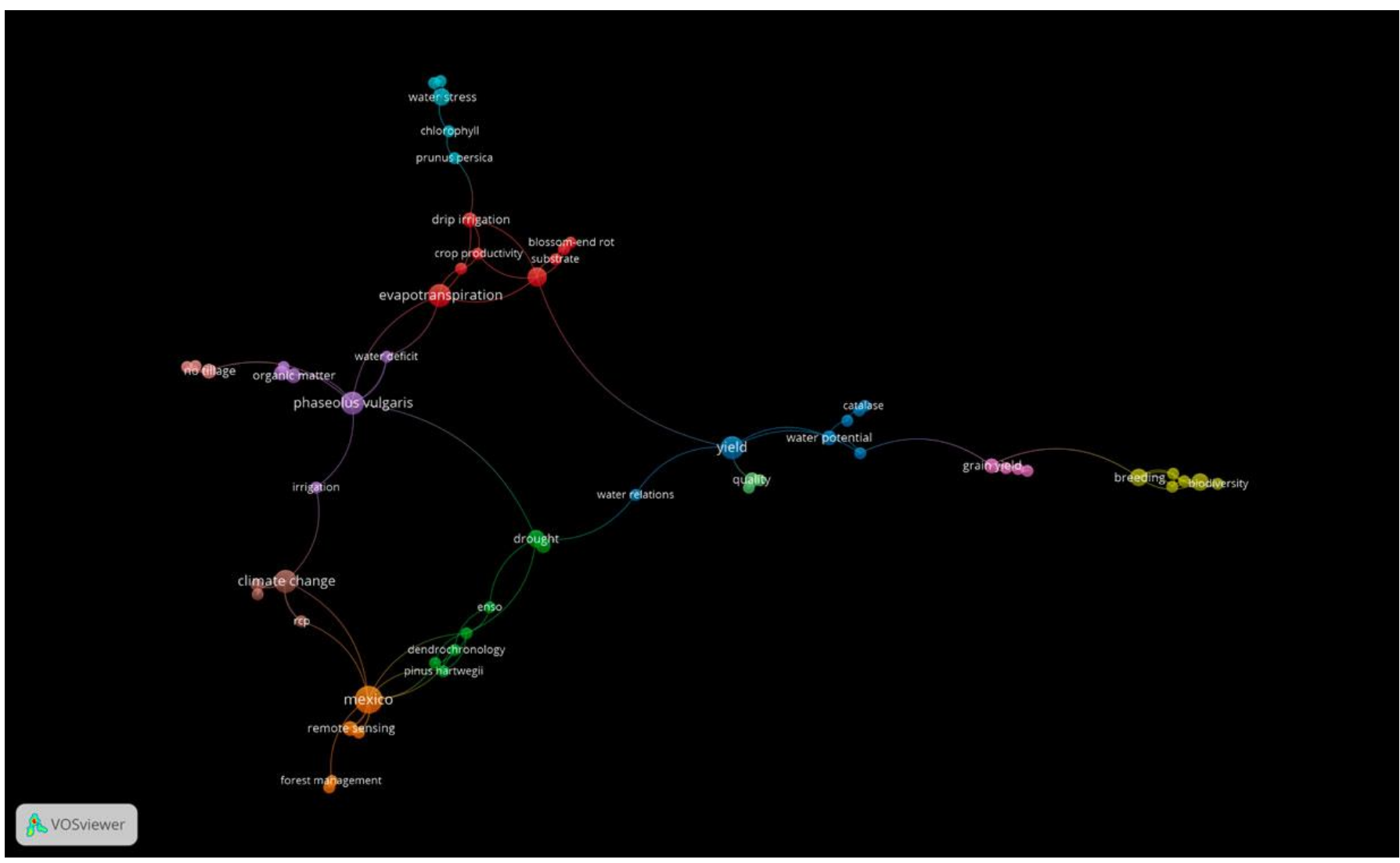

Fuente. Elaboración propia con base en Scopus (2020), creado con VOSviewer.

Figura 4. Red de coocurrencia de palabras clave en la búsqueda delimitada en Scopus a investigadores adscritos a los institutos INIF, INIA, INIP e INIFAP (2000-2020).

La dinámica del incremento de publicaciones a lo largo del período 2002-2020 se muestra en la Figura 5. En 2008, las investigaciones giraron en torno a los rendimientos; en 2010 sobre el uso eficiente del agua; para 2011 sobresalió el tema de evapotranspiración; y recientemente, se incluyó el de cambio climático. 


\section{Trend Topics}

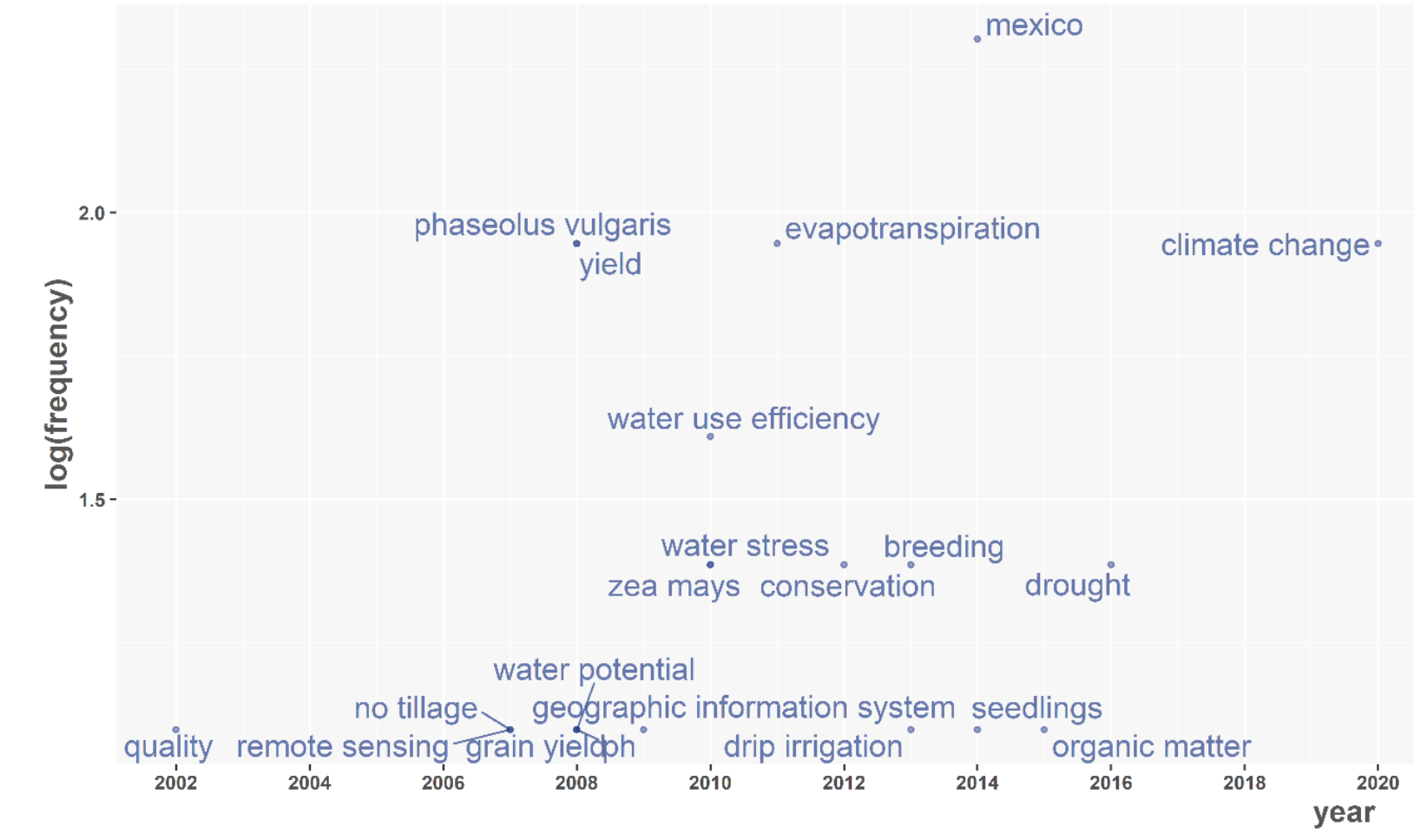

Fuente: Elaboración propia con base en Scopus (2020), creado con Bibliometrix.

Figura 5. Tópicos tendencia en las palabras clave en la búsqueda delimitada de investigadores adscritos al INIFAP en Scopus (2000-2020).

Esta producción documental se aglutinó en 29 revistas y se aprecia que $34.5 \%$ de la producción científica se concentró en 10 revistas mexicanas, en las que se publicaron 74 artículos (66 \%) con autoría de investigadores del INIFAP (Figura 6). No obstante, del total de las revistas, solo nueve incluyeron en su temática alguna referente a los recursos naturales considerados en la presente revisión (suelo, agua, biodiversidad, servicios ambientales y clima), o fueron eminentemente forestales. 


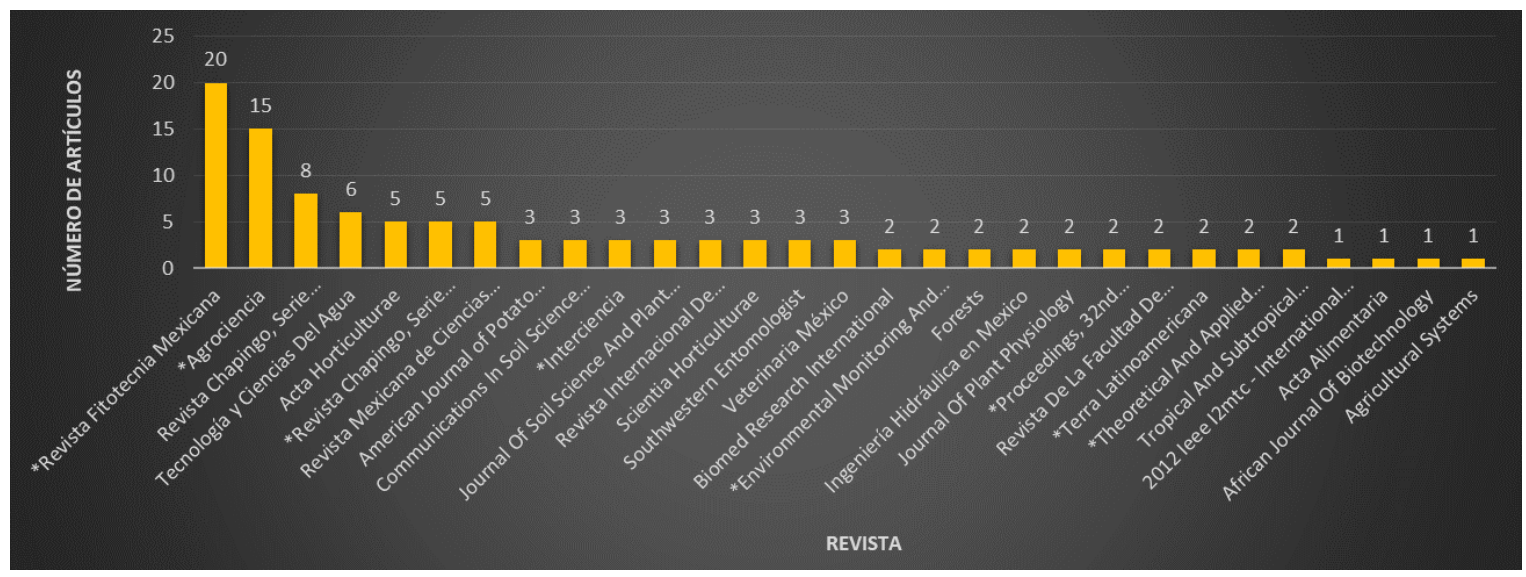

* Revistas cuya temática incluye tópicos de sostenibilidad de los recursos naturales. Fuente. Elaboración propia con base en Scopus (2000-2020).

Figura 6. Revistas indizadas en Scopus donde han publicado los investigadores adscritos al INIFAP sobre temas de sostenibilidad de recursos naturales (2000-2020).

La mayor productividad del INIFAP, con base en el número de artículos publicados, se presenta en los programas de investigación agrícola del INIFAP, cuyos estudios se relacionan con el manejo del agua, el clima, cambio climático, manejo de cuencas (Sánchez-Cohen et al., 2020). Además, se incluyen la domesticación de no maderables (Martínez-Hernández et al., 2017), desarrollo sostenible (Sánchez-Cohen et al., 2008), suelo (Salinas-García et al., 2002; Cruz-Cárdenas et al., 2010) y agricultura sostenible (Acosta-Gallegos et al., 2012). Asimismo, se abordan temas forestales relacionados con el clima, agua y el cambio climático (Villanueva-Díaz et al., 2007, 2018, 2020; Cerano-Paredes et al., 2016).

De los artículos más citados, relacionados con la Sostenibilidad de Recursos Naturales, sobresalen cuatro en los que al menos un autor está adscrito al INIFAP: Muñoz-Villers et al. (2012), con 5.8 citas (total 58); Flores et al. (2006), con 3.5 (total 56); y García-Valenzuela et al. (2005), con 3 (total 51), en este último trabajo todos los autores pertenecen al INIFAP. 
Se identificaron cinco grupos de investigación: el central (color rojo) liderado por SánchezCohen y colaboradores, quienes han sido coautores del grupo de investigación de SifuentesIbarra y López-López (color amarillo), los que a su vez participan en los estudios de Ontiveros-Capurata (investigador del Instituto Mexicano del Agua, IMTA) y colaboradores (color azul) (Figura 7). Sin embargo, el último grupo no tiene relación directa con el de Sánchez-Cohen, cuya centralidad se advierte al identificarse relaciones tanto con el grupo de Cadena-Zapata (color morado), como con el de Villanueva-Díaz (color verde) y Díaz-Padilla (Sánchez-Cohen et al., 2008, 2020; Cerano-Paredes et al., 2009; Díaz-Padilla et al., 2011; Guajardo-Panes et al., 2017). Las redes antes descritas corresponden a investigadores, que si bien, pertenecen al área agrícola, su principal temática de estudio se refiere al suelo (LópezSantos et al., 2012), agua y clima (López-López et al., 2018; Sifuentes-Ibarra et al., 2020).

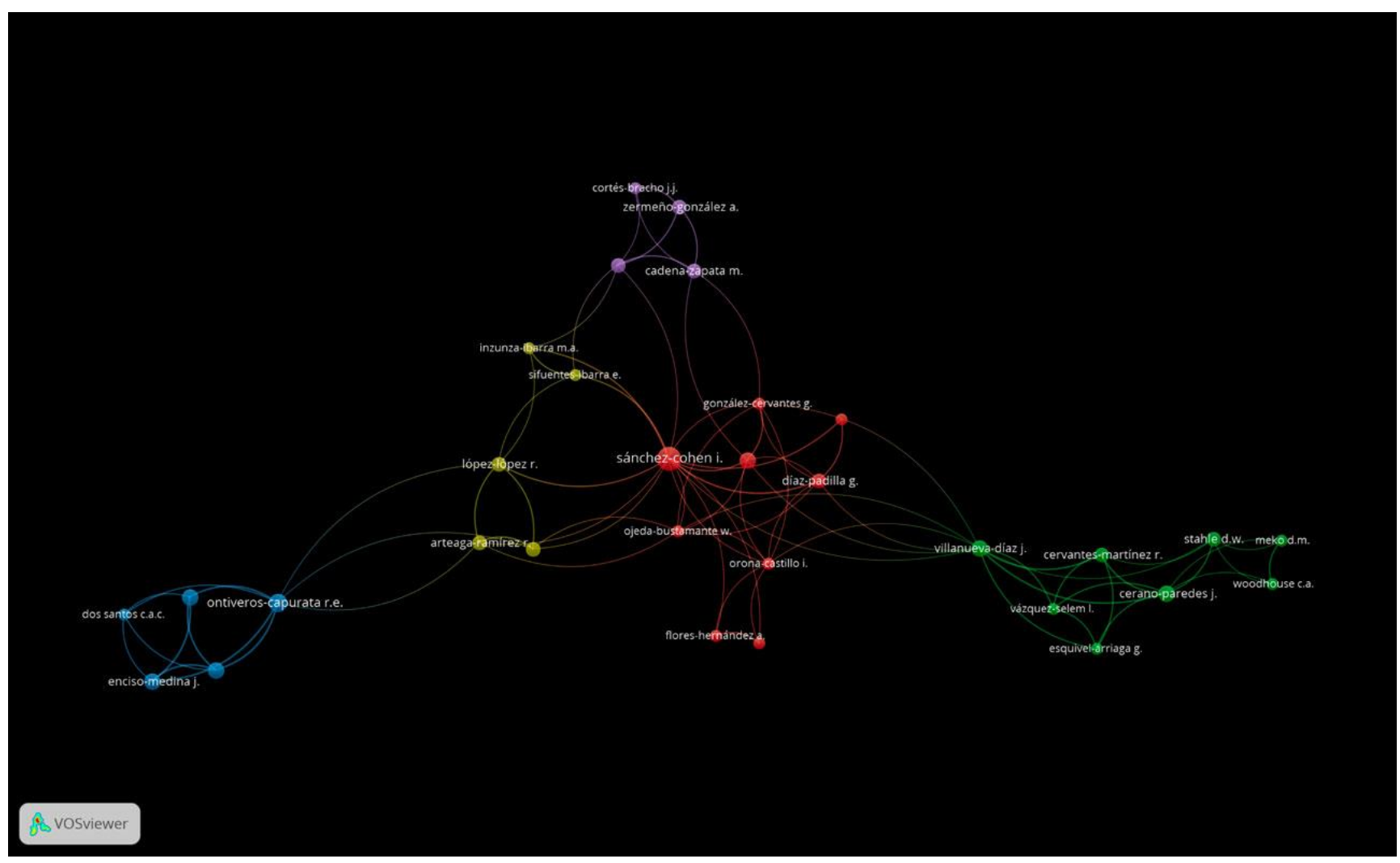

Fuente. Elaboración propia con base en Scopus (2000-2021), creado con VOSviewer.

Figura 7. Red de coautoría de los investigadores adscritos al INIFAP que publican temas sobre "Sostenibilidad de Recursos Naturales" en revistas indizadas en Scopus. 
Cabe señalar que el grupo central (Sánchez-Cohen et al.) también mantiene colaboraciones con investigadores del extranjero (Sánchez-Cohen et al., 2006). Tanto Sánchez-Cohen como Villanueva-Díaz son investigadores adscritos del Centro Nacional de Investigación Disciplinaria en la Relación, Agua, Suelo, Planta y Atmósfera (CENID-RASPA), donde se abordan aspectos fundamentales para la sostenibilidad de los recursos naturales (suelo, agua, clima); además, se advierte que el compartir un espacio físico facilita la interrelación laboral, lo cual se evidencia con la conexión de coautoría entre Villanueva-Díaz y Cerano-Paredes (Figura 7).

\section{Revisión documental en la sub-base Scielo Citation Index de Web of Science (2002 a 2020)}

Respecto a la red de coocurrencia de palabras clave, se observó que tanto sequía, como cambio climático fueron los nodos centrales; también sobresalieron los referentes a manejo forestal, plantaciones forestales e inventario forestal (Figura 8). Es decir, fueron coincidentes con los resultados de la revisión correspondiente a la base de datos de Scopus (Figura 3), aun cuando esta es más global, ya que su acervo comprende publicaciones de todo el mundo; en cambio, la revisión de WoS se circunscribió a la sub-base conformada por revistas editadas en Iberoamérica. 


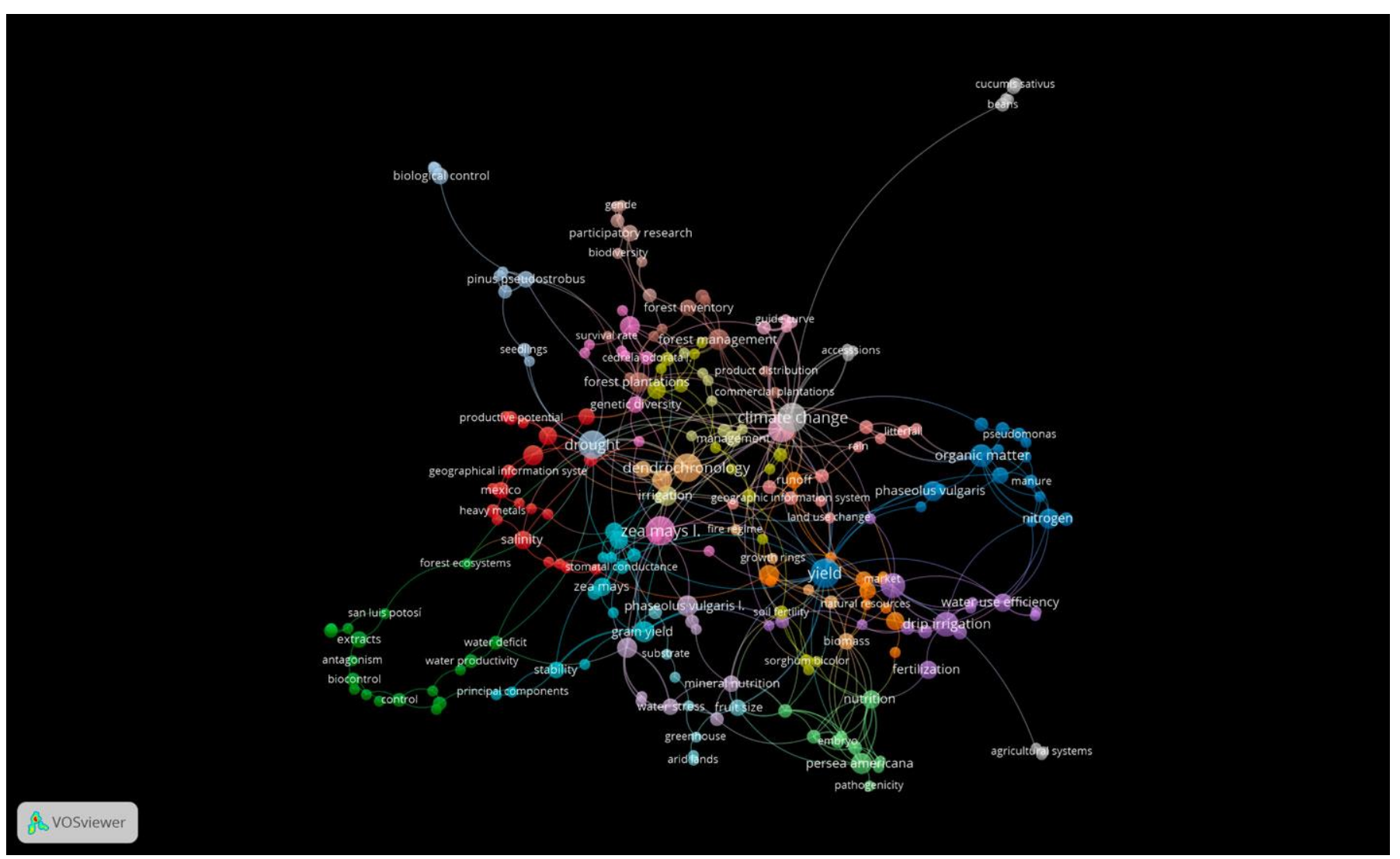

Fuente. Elaboración propia con base en SciELO Citation Index (2002-2020) creado con VOSviewer.

Figura 8 Red de coocurrencia de la búsqueda de artículos indizados de investigadores adscritos al INIFAP sobre sostenibilidad de recursos naturales en SciELO Citation Index (2002-2020).

La dinámica de los tópicos tendencia tuvo su despunte a partir de 2009 con cambio climático, en tanto que en 2012, los temas de interés fueron productividad y sequía; finalmente; en los últimos cuatro años empezaron a destacar manejo forestal, plantaciones forestales y conservación (Figura 9). 


\section{Trend Topics}

2.6-

climate change.

$$
\begin{aligned}
& \text { yield .zea mays I. } \\
& \text { dendrochronology } \\
& \text { drought }
\end{aligned}
$$

$2.4-$

$$
\text { growth }
$$

drip irrigation

evapotranspiration

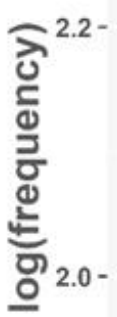

organic matter

grain yield phaseolus vulgaris I.

persea americana

forest management•

1.8- water balance irrigation phaseolus vulgaris çonservation nitrogen' water potential remote sensing enso forest plantations

fruit quality

1.6- salinity soil moisture nutrition geographic information systems 2009 forti $\quad 2013 \quad 2015 \quad 2017$ year

Fuente. Elaboración propia con base en Scielo Citation Index (20022020), creado con Bibliometrix.

Figura 9. Tópicos tendencia en las palabras clave en la búsqueda sobre sostenibilidad de recursos naturales indizada en Scielo Citation Index de las publicaciones de investigadores adscritos al INIFAP. 
Por otra parte, se registraron 32 revistas (Figura 10) en las que frecuentemente publican los investigadores del INIFAP, entre las que destacaron aquellas con temas referentes a la Sostenibilidad de los Recursos Naturales. Entre ellas, 17 incluyen menos de cinco artículos, pero algunas con temática forestal, o bien de aspectos referentes a la biodiversidad vegetal: Acta Botánica (4 artículos), Revista Mexicana de Biodiversidad (3), Polibotánica (3) y Bosque (Valdivia, Chile) (2).

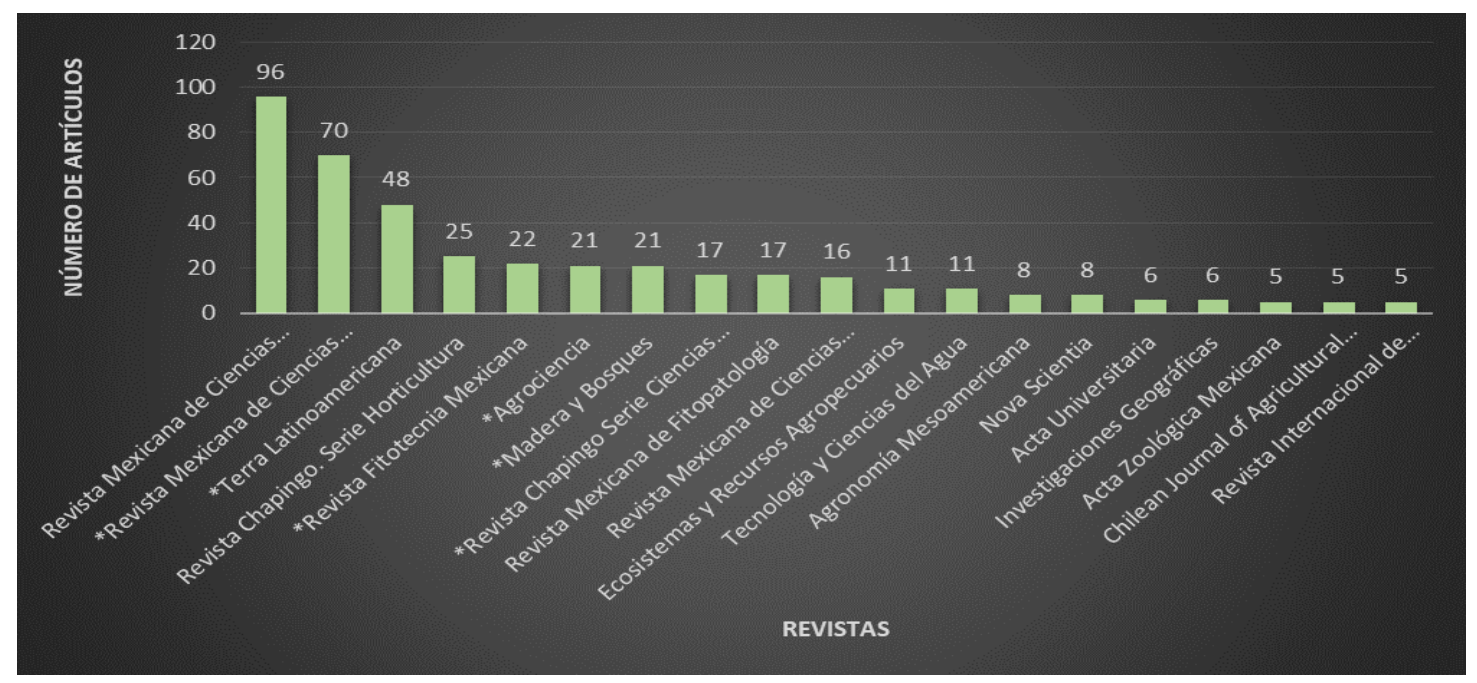

Fuente. Elaboración propia con base en Scielo Citation Index (2002-2021). *Revistas con temática forestal.

Figura 10. Revistas indizadas en Scielo Citation Index en las que publican sobre Sostenibilidad de Recursos Naturales los investigadores adscritos al INIFAP.

Las publicaciones con más de 10 artículos son editadas en México y, de estas, resaltaron las del INIFAP: Revista Mexicana de Ciencias Agrícolas (96 artículos) y Revista Mexicana de Ciencias Forestales (70) (Figura 9). Es decir, el personal científico del INIFAP prefiere dar a conocer sus resultados de investigación en revistas nacionales, particularmente, en las de su propia institución. Lo anterior coincide con lo citado en la literatura para temas como modelación forestal, cuya producción 
científica en México, durante el período 1980-2015, se publicó en 31 revistas mexicanas, de un total de 37 identificadas (Martínez-Santiago et al., 2017).

Se identificó una red de colaboración entre 15 grupos y subgrupos de investigación. De ellos, seis compartieron cierta centralidad en la red de coautorías. De nuevo, destacaron los grupos de investigación de Ignacio Sánchez-Cohen y José VillanuevaDíaz; cabe señalar que ambos tuvieron centralidad en la red de coautorías elaborada con los datos de Scopus. Sin embargo, en este caso resalta la presencia de subgrupos que no se mostraban en las redes construidas a partir de la información de esa plataforma; entre ellos, aquéllos en los que participan Julián Cerano-Paredes, Miguel Acosta-Mireles, Antonio González Hernández, Jonathan Hernández-Ramos y José Ariel Ruiz-Corral, aunque este último adscrito a la Universidad de Guadalajara (Figura 11).

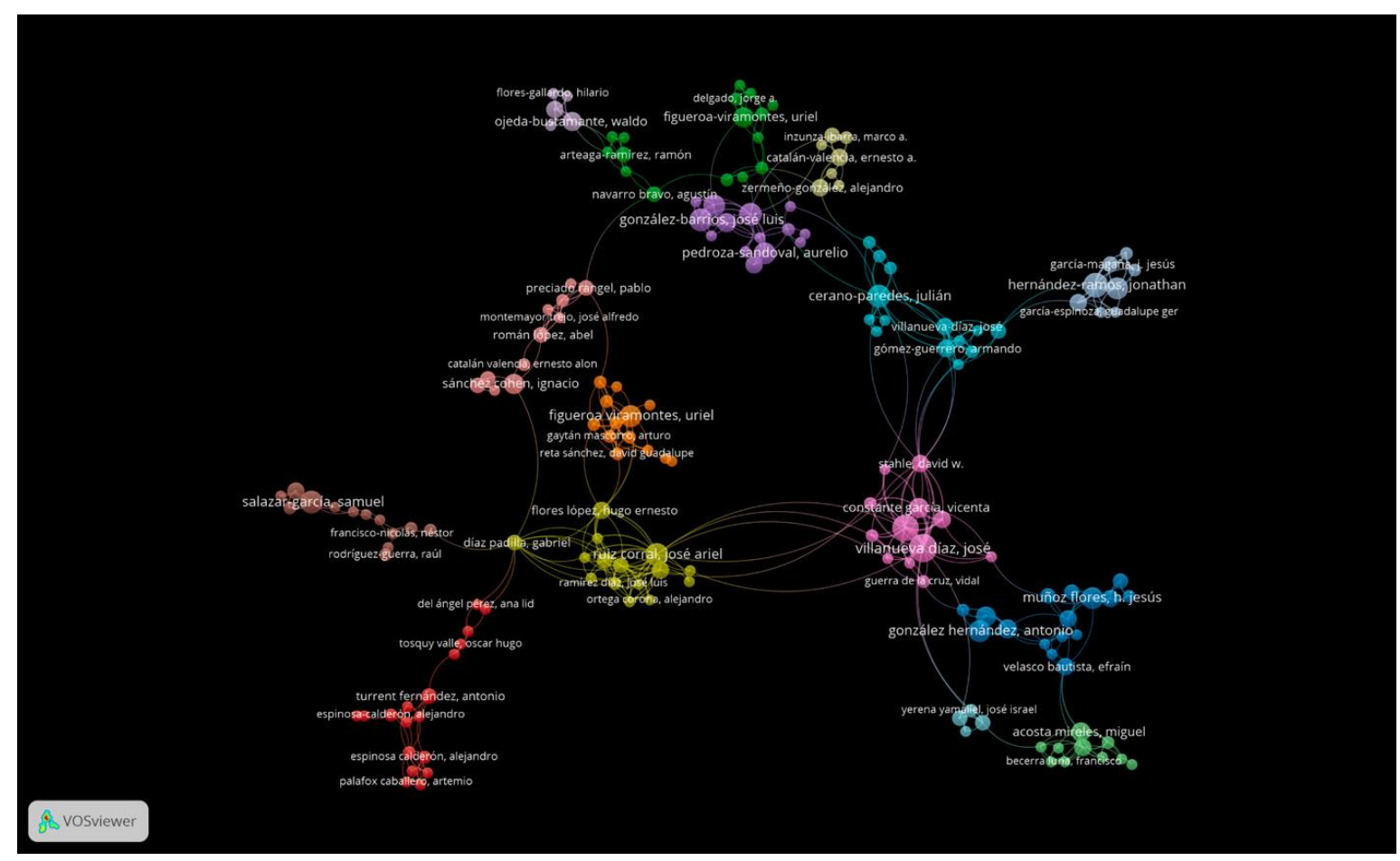

Fuente. Elaboración propia con base en SciELO Citation Index (2002-2020), creado con VOSviewer.

Figura 11. Red de coautoría de los autores adscritos al INIFAP con publicaciones sobre Sostenibilidad de Recursos Naturales indizadas en SciELO Citation Index. 
Respecto a las redes de coautoría entre investigadores adscritos al INIFAP con los de otras instituciones, se identificó una colaboración estrecha con 13 organizaciones, principalmente nacionales, tal es el caso de la Universidad Autónoma Chapingo y la Universidad Autónoma Agraria Antonio Narro. Además, existe cierta interrelación con El Colegio de la Frontera Sur y las Universidades Autónomas de Nayarit, Nuevo León, Chihuahua, Guadalajara y el Tecnológico de Sonora entre otras (Figura 11).

En este punto, es necesario recomendar que se implemente una política institucional que promueva entre los investigadores el uso de un nombre normalizado de la institución, ya que se utiliza tanto INIFAP, como Instituto Nacional de Investigaciones Agrícolas y Pecuarias (Figura 12), lo que incide en una pérdida de registros de la producción académica asociada a la institución.

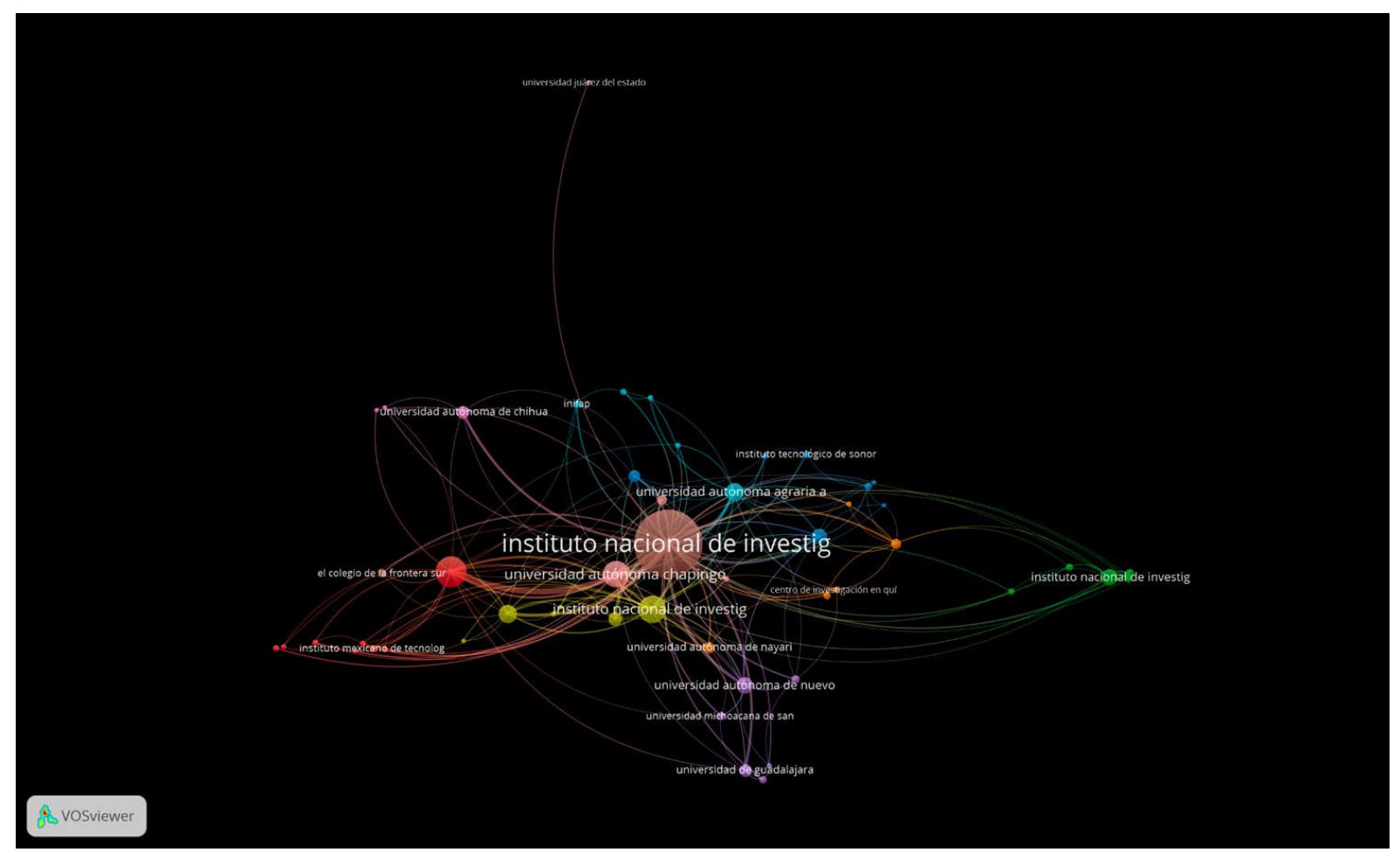

Fuente. Elaboración propia con base en SciELO Citation Index (2002-2020), creado con VOSviewer.

Figura 12. Red de coautoría por institución, con quienes autores adscritos al INIFAP sostienen colaboración, en los artículos sobre Sostenibilidad de Recursos Naturales indizados en SciELO Citation Index. 
Las redes de colaboración constituyen una herramienta para conocer los nexos académicos y de trabajo inter e intrainstitucionales (Huamaní y Mayta-Tristan, 2010). Cuando estas redes se consolidan, facilitan el acceso a los recursos económicos ofertados por las instancias financiadoras de la investigación, a través de la integración, por ejemplo, de consorcios capaces de atender problemas a gran escala, como los de nivel regional o país (Gaughan y Ponomariov, 2008; Huamaní y Mayta-Tristán, 2010). En México, este tipo de análisis es común en estudios sociales, bibliométricos y en el manejo de recursos (Calderón y Flores, 2012; Núñez-Espinoza et al., 2014; Martínez et al., 2015; Martínez-Santiago et al., 2017; Gallardo-Salazar et al., 2020).

\section{Revisión documental de la Revista Mexicana de Ciencias Forestales (1985 a 2009)}

La tendencia en este período hacia la publicación de artículos relacionados con la Sostenibilidad de los Recursos, en el caso de los programas actuales de investigación del INIFAP, fue que la mayoría de los autores pertenecieron al de Manejo Forestal Sustentable con 40.9 \% de los artículos (Moreno, 1988; Parraguirre, 1993; Moreno y Moreno, 1995; García et al., 1993; Sotelo et al., 2005; Mallén, 2008; Ortíz et al., 2008) y Plantaciones Forestales con 31.8 \% (Muñoz et al., 2009; Rodríguez et al., 2004). Ambos programas tienen líneas de investigación cuyos productos inciden en la sustentabilidad de los ecosistemas forestales (Figura 13). 


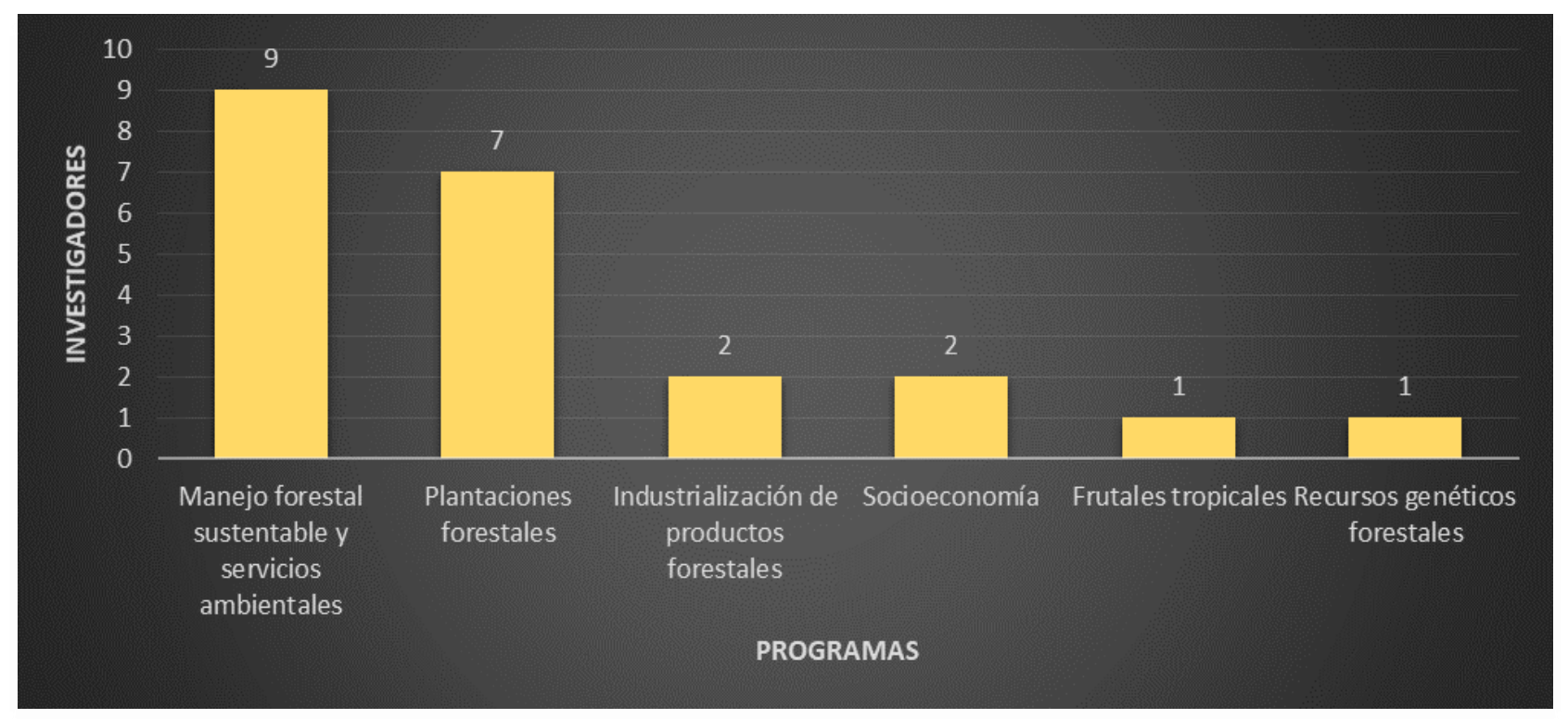

Figura 13. Investigadores del INIFAP por programa de investigación que publicaron sobre Sostenibilidad de los Recursos Naturales en la Revista Mexicana de Ciencias Forestales (1985-2009).

De los artículos publicados en la RMCF sobre la Sostenibilidad de los Recursos Naturales, se identificaron 14 adscripciones de los autores participantes, de los cuales en $78.6 \%$ de los artículos hay presencia de investigadores del INIFAP; mientras que, el Colegio de Postgraduados y la Universidad Autónoma de Chapingo están en $10.7 \%$, cada uno (Figura 14). El resto de las instituciones solo se presentaron en una ocasión. 


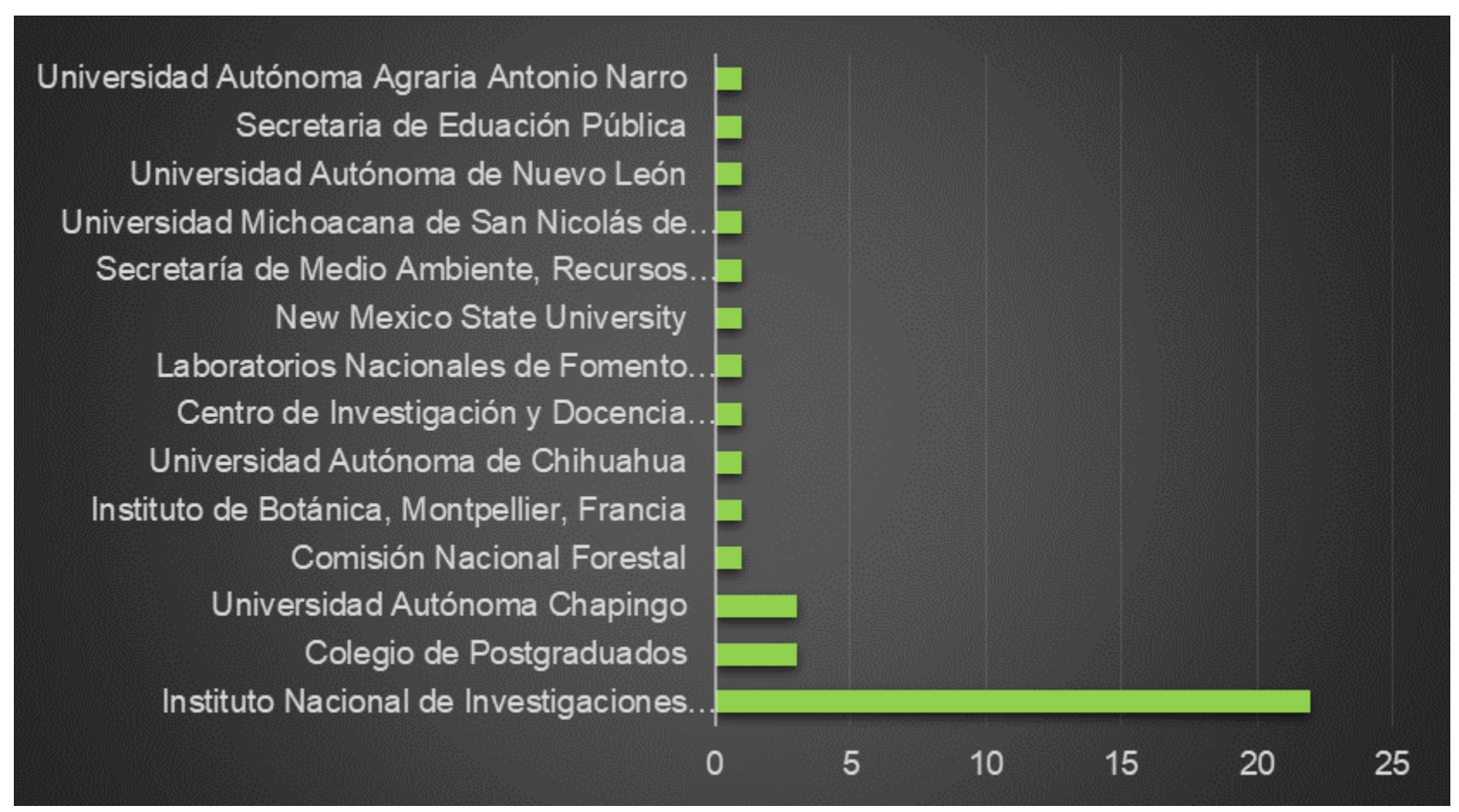

Figura 14. Participación por centro de adscripción en artículos relacionados con la Sostenibilidad de los Recursos Naturales publicados en la Revista Mexicana de Ciencias Forestales en el período 1985-2009.

La mayor parte de los trabajos estuvieron dirigidos hacia la sostenibilidad de los recursos maderables (78.6\% de los artículos registrados y $68.8 \%$ en frecuencia de aparición); por ejemplo: Juárez y Ramírez (1985); Moreno (1988); García et al. (1993). Durante ese período iniciaba el interés por publicar artículos sobre los recursos no maderables y el suelo.

Finalmente, la producción de los investigadores del INIFAP en lo que se denomina "literatura gris" es cuantiosa (Martínez-Méndez y López-Carreño 2011); es decir, documentos como Folletos, Manuales y Publicaciones especiales, (https://vun.inifap.gob.mx/BibliotecaWeb/_Content); no obstante dicha producción queda fuera de la revisión que aquí se describe. 


\section{Las investigaciones forestales sobre sostenibilidad de los recursos naturales en el INIFAP y sus perspectivas}

En el proceso de consolidación del INIFAP, sus programas de investigación han estado sujetos a una mejora continua en respuesta a las demandas de sus usuarios y de los problemas ambientales que inciden directa o indirectamente sobre el capital natural del país; los cuales, a su vez, inciden tanto en las políticas nacionales de investigación, como en las estrategias de financiamiento a la investigación.

En particular, en los últimos 10 años han adquirido relevancia los temas referentes a los servicios ecosistémicos, el cambio climático y el manejo forestal multipropósito; este último referido a su impacto y coexistencia con el aprovechamiento y conservación de la biodiversidad (Monárrez-González et al., 2018; Zamora et al., 2018; Chávez-León, 2019). Asimismo, el INIFAP ha dado respuesta a la demanda de información de base científica requerida para entender cómo afecta el cambio climático a los bosques, entendidos estos como áreas forestales, desde la perspectiva del desarrollo de las masas forestales, los incendios forestales, la permanencia de los cuerpos de agua, la captura y almacenamiento de agua en superficies forestadas.

En el contexto hidrológico forestal, además de su relevancia en el esquema del Pago por Servicios Ambientales, es importante el trabajo interdisciplinario bajo el enfoque del manejo integral de cuencas, para lo cual son relevantes las experiencias de investigadores líderes en la investigación del agua en el INIFAP (Sánchez-Cohen et al., 2008; López-Báez, 2014). Lo anterior, sin dejar de lado la conformación de redes de colaboración con instituciones afines que abordan temas sobre este recurso: Instituto Mexicano del Agua (IMTA), el Colegio de Postgraduados, Universidad Autónoma Chapingo, Instituto Potosino de Ciencia y Tecnología, entre otros.

Respecto al incremento del número de publicaciones de investigadores forestales, en los últimos quince años el INIFAP generó un programa de reclutamiento y selección de profesionistas, con la intención de iniciar el cambio generacional de su personal científico. Ello incidió positivamente en el área Forestal del Instituto, ya que permitió reforzar el 
desarrollo de la investigación en temas específicos dentro de los programas institucionales; facilitó el fortalecimiento de equipos de trabajo y el incremento de su productividad.

En el caso de México, es prioritario continuar con los esfuerzos de conservación del capital natural del país para lograr la sustentabilidad de los ecosistemas y, mediante la integración de más redes de colaboración inter e intrainstitucionales, desarrollar estudios de sostenibilidad que coadyuven a la generación de una línea base de conocimientos enfocados al desarrollo sostenible de los ecosistemas.

En el contexto del desarrollo sostenible, un aspecto pendiente en el INIFAP es la conformación de colaboraciones multisectoriales, a partir de la fortaleza institucional que representa su capital humano con experiencia y conocimientos en los sectores forestal, agrícola y pecuario, además de un grupo de investigadores dedicados a la ejecución de estudios socioeconómicos; lo cual constituye un gran potencial para la realización de proyectos de gran visión sobre el desarrollo sostenible de los recursos naturales.

Finalmente, es necesario favorecer la visibilidad de los conocimientos generados en el INIFAP con la promoción de su publicación en revistas nacionales no institucionales e, incluso, en las editadas fuera de México, pero sobre todo en revistas incluidas en índices bibliométricos de impacto internacional.

\section{Conflicto de intereses}

Marisela Cristina Zamora-Martínez declara no haber participado en el proceso editorial del presente manuscrito, ya que forma parte del Comité Editorial de la Revista Mexicana de Ciencias Forestales. 


\section{Contribución por autor}

Marisela Cristina Zamora-Martínez: diseño, estructura, revisión bibliográfica, redacción y corrección del documento; Aixchel Maya-Martínez y Nelda Guadalupe Uzcanga-Pérez: revisión bibliográfica, análisis de la información de la Revista Mexicana de Ciencias Forestales (1985-2009), redacción y revisión del documento; Rosario Rogel-Salazar e Irvin Santiago-Bautista: análisis de la información de Scopus y WoS, revisión del documento; Guadalupe Fabiola Reygadas Prado: compilación bibliográfica, redacción y revisión del documento; Eulalia Edith VillavicencioGutiérrez: compilación bibliográfica, redacción y revisión del documento.

\section{Referencias}

Acosta-Gallegos, J. A., E. Espinoza-Trujillo, B. M. Sanchez-Garcia, Y. JiménezHernández, R. A. Salinas-Perez, R. Rosales-Serna, R: Zandate-Hernández and C. González-Rivas. 2012. Adaptability of the flor de junio dry bean type to different environments in Mexico. Tropical and Subtropical Agroecosystems 15(2): 427-438. https://www.scopus.com/inward/record.uri?eid=2-s2.0-

84868131078\&partnerID=40\&md5=bf4a7c07a4969549a006a3100c7e617a ( 1 de marzo de 2021).

Aria, M. and C. Cuccurullo. 2017. Bibliometrix: An R-tool for comprehensive science mapping analysis. Journal of Informetrics 11(4): 959-975.

Doi:10.1016/j.joi.2017.08.007.

Arnold, M., B. Powell, P. Shanley and T.C.H. Sunderland. 2011. Forests, biodiversity and food security. International Forestry Review 13(3): 259-264.

Doi:10.1505/146554811798293962?journalCode=ifre.

Barrios N., C., U. Castro A., G. Coria L., M. González A., R. Martínez V. y L. Taddey D. 2007. La relación global-local. Sus implicancias prácticas para el diseño de estrategias de desarrollo. Red Académica Iberoamericana Local-Global-EUMEDNET. www.eumed.net/libros/2007a/259/index.htm (15 de marzo de 2021). 
Barton, J. R. and F. A. Gutiérrez-Antinopai. 2020. Towards a Visual Typology of Sustainability and Sustainable Development. Sustainability 12(19):7935. Doi: 10.3390/su12197935.

Calderón M., M. G. y J. Flores P. 2012. Redes de conocimiento en empresas de la industria electrónica en México: Una propuesta metodológica. Economía: teoría y práctica 37: 121-143. http://www.scielo. org.mx/scielo.php?script=sci_arttext\&pid=S0188$33802012000200006 \&$ lng=es\&tlng=es ( 2 de marzo de 2021).

Cerano-Paredes, J., J. Villanueva-Díaz, J. G. Arreola-Ávilaz, I. Sánchez-Cohen, Y. R. D. Valdez-Cepeda and G. García-Herrera. 2009. Reconstructing 350 years of precipitation in southeast Chihuahua, Mexico. Madera Bosques 15(2): 27-44. Doi: $10.21829 /$ myb.2009.1521189.

Cerano-Paredes, J., J. Villanueva-Díaz, L. Vázquez-Selem, R. Cervantes-Martínez, G. Esquivel-Arriaga, V. Guerra-de la Cruz and P. Z. Fulé. 2016. Historical fire regime and its relationship with climate in a forest of Pinus hartwegii to the north of Puebla State, Mexico. Bosque 37(2): 389-399. Doi:10.4067/S0717-92002016000200017.

Chávez-León, G. 2019. Diversidad de mamíferos y aves en bosques de coníferas bajo manejo en el Eje Neovolcánico Transversal. Revista Mexicana de Ciencias Forestales 10(56):85-112. Doi:10.29298/rmcf.v10i56.499.

Chopra, K., R. Leemans, K. Pushpam and S. Henk (eds.). 2005. Ecosystems and Human Well-being: Policy Responses: findings of the Responses Working Group of the Millennium Ecosystem Assessment Volume 3. Island Press. Washington, DC, USA. pp. 2-21.

Comisión Nacional para el Conocimiento y Uso de la Biodiversidad (Conabio). 2008. Capital natural de México, vol. I: Conocimiento actual de la biodiversidad. Comisión Nacional para el Conocimiento y Uso de la Biodiversidad. México, D. F., México. 621 p. www2.biodiversidad.gob.mx/país/pdf/CapNatMex/Vol\%201/100_PrefasioGuia.pdf. (2 de enero de 2021). 
Cruz-Cárdenas, G., C. A. Ortiz-Solorio, E. Ojeda-Trejo, J. F. Martínez-Montoya, E. D. Sotelo-Ruiz and A. L. Licona-Vargas. 2010. Digital mapping of farmland classes in three landscapes in Mexico. Journal of Soil Science and Plant Nutrition 10(4): 414427. Doi: $10.4067 /$ S0718-95162010000200003.

Díaz-Padilla, G., I. Sánchez-Cohen, R. Quiroz, J. Garatuza-Payán, C. Watts-Thorp, I. R. CruzMedina and R. A. Guajardo-Panes. 2011. Space-time variation of rainfall in Mexico: An approach for assessing impacts. Tecnología y Ciencias del Agua 2(4):51-64. https://www.imta.gob.mx/revistadigital198237645imta_comunicacion/rev-04-2011/files/. (15 de febrero de 2021).

Flores, J., E. Jurado and A. Arredondo. 2006. Effect of light on germination of seeds of Cactaceae from the Chihuahuan Desert, Mexico. Seed Science Research 16(2): 149-155. Doi: 10.1079/SSR2006242.

Gallardo-Salazar, J. L., M. Pompa-García, C. A. Aguirre-Salado, P. M. López-Serrano y A. Meléndez-Soto. 2020. Drones: tecnología con futuro promisorio en la gestión forestal. Revista Mexicana de Ciencias Forestales 11(61): 27-50.

Doi:10.29298/rmcf.v11i61.794.

García C., X., B. Rodríguez S. y C. Parraguirre L. 1993. Notas importantes sobre el chicozapote (Manilkara zapota L. Van Royen). Ciencia Forestal en México 18(74):46-63. https://cienciasforestales.inifap.gob.mx/editorial/index.php/forestales/article/view/1 048 (5 de enero de 2021).

García-Valenzuela, X., E. Garcá-Moya, Q. Rascón-Cruz, L. Herrera-Estrella and G. A. AguadoSantacruz. 2005. Chlorophyll accumulation is enhanced by osmotic stress in graminaceous chlorophyllic cells. Journal of Plant Physiology 162(6):650-61. Doi: 10.1016/j.jplph.2004.09.015. 
Gaughan, M. and B. Ponomariov. 2008. Faculty publication productivity, collaboration, and grants velocity: Using curricula vitae to compare center-affiliated and unaffiliated scientists. Research Evaluation 17(2): 103-10. Doi: 10.3152/095820208X287180.

Guajardo-Panes, R. A., G. R. Granados-Ramírez, I. Sánchez-Cohen, G. Díaz-Padilla and F. Barbosa-Moreno. 2017. Spatial validation of climatological data and homogeneity tests: The case of Veracruz, Mexico. Tecnología y Ciencias del Agua 8(5): 157-177. Doi:10.24850/j-tyca-2017-05-11.

Huamaní, C. y P. Mayta-Tristán. 2010. Producción científica peruana en medicina y redes de colaboración, análisis del Science Citation Index 2000-2009. Revista Peruana de Medicina Experimental y Salud Pública 27(3): 315-325. http://www.scielo.org.pe/pdf/rins/ v27n3/a03v27n3.pdf (16 de marzo de 2021). International Business Machines (IBM) 2015. IBM SPSS Statistics para Windows, versión 23.0. IBM Corp. Armonk, NY, USA. n/p.

Instituto Nacional de Investigaciones Forestales, Agrícolas y Pecuarias (INIFAP). 2018. Programa de desarrollo del INIFAP 2018-2030. INIFAP. Ciudad de México, México. 456 p.

Juárez G., V. y H. Ramírez M., H. 1985. Crecimiento de Gmelina arborea (L.) Roxb. en cuatro espaciamientos. Ciencia Forestal 10 (56): 33-45.

Kuhlman, T. and J. Farrington. 2010. What is sustainability? Sustainability 2(11): 3436-3448. Doi:10.3390/su2113436.

López R., C. D., E. S. López H. y P. I. Ancona. 2015. Desarrollo sustentable o sostenible: una definición conceptual. Horizonte Sanitario (4)2: 1-7. http://www.redalyc.org/pdf/4578/457845044002.pdf (12 de febrero de 2021). 
López-Báez, W. 2014. Análisis del manejo de cuencas como herramienta para el aprovechamiento sustentable de recursos naturales. Revista Chapingo Serie Zonas Áridas 13(2): 39-45. https://www.redalyc.org/articulo.oa?id=455545055001 (16 de marzo de 2021).

López-Santos, A., G. González-Cervantes, M. Cadena-Zapata and J. L. GonzálezBarrios. 2012. Effect of primary tillage on the physical quality of soil, as evaluated by disk permeameter. Tecnología y Ciencias del Agua 3(4): 127-141.

https://www.scopus.com/inward/record.uri?eid=2-s2.0-

84880422022\&partnerID $=40 \&$ md5 $=72$ b84c93566190e7e563194d7a916a00 (6 de febrero de 2021).

López-López, R., J. A. Jiménez-Chong, I. Hernández-Aragón and M. A. Inzunza Ibarra. 2018. Water productivity of rice genotypes with irrigation and drainage. Irrigation and Dainage 67: 508-515. Doi: 10.1002/ird.2250.

Mallén R., C. 2008. Indicadores ambientales: la evaluación de los recursos naturales. Ciencia Forestal en México 33(104):155-190.

https://cienciasforestales.gob.mx/editorial/index.php/forestales/article/view/731 (12 de febrero de 2021).

Martínez-Hernández, R., M. Villa-Castorena, E. A. Catalán-Valencia and M. A. Inzunza-Ibarra 2017. Production of oregano (Lippia graveolens Kunth) seedling from seeds in nursery for transplanting. Revista Chapingo, Serie Ciencias Forestales y del Ambiente 23(1):61-73. Doi:10.5154/r.rchscfa.2015.11.051.

Martínez-Méndez, F. J. y R. López-Carreño. 2011. El sinsentido de hablar de literatura gris en la época 2.0. El Profesional de la Información 20(6): 621-626. Doi:10.3145/epi.2011.nov.03. 
Martínez-Santiago, S., A. Alvarado-Segura, F. J. Zamudio-Sánchez and D. CristóbalAcevedo. 2017. Spatio-temporal analysis of forest modeling in Mexico. Revista Chapingo Serie Ciencias Forestales y del Ambiente 23: 5-22.

Doi: 10.5154/r.rchscfa.2016.01.003.

Martínez, N., L. Brenner e I. Espejel. 2015. Red de participación institucional en las áreas naturales protegidas de la península de Baja California. Región y sociedad 27(62): 27-62. http://www.scielo.org.mx/scielo.php?script=sci_

arttext\&pid=S1870-39252015000100002\&lng=es\&tln g=es (5 de febrero de 2021).

Monárrez-González, J. C., G. Pérez-Verdín, C. López-González, M. A. MárquezLinares y M. S. González-Elizondo. 2018. Efecto del manejo forestal sobre algunos servicios ecosistémicos en los bosques templados de México. Madera y Bosques 24(2):e2421569. Doi:10.21829/myb.2018.2421569.

Moreno S., R. 1988. Modelo de programación por objetivos para la plantación de un sistema de abastecimiento forestal. Ciencia Forestal 13(63):175-190. https://cienciasforestales.inifap.gob.mx/ (5 de enero de 2021).

Moreno S., R. y F. Moreno S. 1995. Los sistemas de información geográfica en la administración de recursos naturales: recomendaciones de las experiencias del INIFAP. Ciencia Forestal en México 20(78):93-109.

http://cienciasforestales.inifap.gob.mx/editorial/index.php/forestales/article/view/1011 (7 de enero de 2021).

Muñoz F., H. J., V. M. Coria Á., J. J. García S. y M. Balam C. 2009. Evaluación de una plantación de tres especies tropicales de rápido crecimiento en Nuevo Urecho, Michoacán. Ciencia Forestal en México 34(106):61-87.

https://cienciasforestales.inifap.gob.mx/editorial/index.php/forestales/article/view/6 84/1691 (8 de enero de 2021). 
Muñoz-Villers, L. E., F. Holwerda, M. Gómez-Cárdenas, M. Equihua, H. Asbjornsen, L. A. Bruijnzeel, B. E. Marín-Castro and C. Tobón. 2012. Water balances of oldgrowth and regenerating montane cloud forests in central Veracruz, Mexico. Journal of Hydrology 462-463: 53-66. Doi: 10.1016/j.jhydrol.2011.01.062.

Núñez-Espinoza, J. F., O. L. Figueroa R. y L. Jiménez-Sánchez. 2014. Elementos para analizar redes sociales para el desarrollo rural en México: El caso RENDRUS. Agricultura, sociedad y desarrollo 11(1): 1-24.

http://www.scielo.org.mx/scielo.php?script=sci_arttext\&pid=S1870-

$54722014000100001 \& \operatorname{lng}=e s \& \ln \mathrm{g}=\mathrm{es}$ ( 8 de febrero de 2021).

Organización de las Naciones Unidas (ONU). 1987. Nuestro futuro común. Informe Brundtland. Comisión Mundial sobre el Medio Ambiente y el Desarrollo. Organización de las Naciones Unidas. http://www.un.org/es/comun/docs/?symbol=A/42/427 (5 de febrero de 2021).

Organización de las Naciones Unidas para la Alimentación y la Agricultura (FAO). 1992. Conferencia de las Naciones Unidas sobre el Medio Ambiente y el Desarrollo (CNUMAD). Principios Forestales, capítulo 11 de la Agenda 21

https://www.un.org/esa/dsd/agenda21_spanish/res_riodecl.shtml (8 febrero de 2021).

Ortíz T., C., F. Camacho M., E. Flores A. y P. de la Garza L. L. 2008. Potencial productivo en el Distrito Federal para árboles de Navidad y arbustos de uso múltiple. Ciencias Forestal en México 33(103):103-28.

https://cienciasforestales.gob.mx/editorial/index.php/forestales/article/view/743. (5 de febrero de 2021).

Parraguirre L., C. 1993. Métodos de enriquecimiento de las selvas de Quintana Roo. Ciencia Forestal en México. 18(74): 65-79.

https://cienciasforestales.inifap.gob.mx/editorial/index.php/forestales/article/view/1 049 (5 de febrero de 2021). 
Rivera-Hernández, J. E., G. Alcántara-Salinas, N. V. Blanco-Orozco, E. Pascal H. y J. A. Pérez-Sato. 2017. ¿Desarrollo sostenible o sustentable? La controversia de un concepto. Revista Posgrado y Sociedad 15(1): 57-67. Doi:10.22458/rpys.v15i1.1825.

Rodríguez F., F. J., J. Á. Prieto R. y J. J. Návar C. 2004. Producción de biomasa en plantaciones forestales del norte de México. Ciencia Forestal en México 29(96): 67-90.

https://cienciasforestales.gob.mx/editorial/index.php/forestales/article/view/870 (8 de febrero de 2021).

Salas-Zapata, W., L. Rios-Osorio and J. Cardona-Arias. 2017. Methodological characteristics of sustainability science: A systematic review. Environment, Development and Sustainability 19(4): 1127-1140. Doi:10.1007/s10668-016-9801-z.

Salinas-García, J. R., J. D. J. Velázquez-García, M. Gallardo-Valdez, P. DíazMederos, F. Caballero-Hernández, L. M. Tapia-Vargas and E. Rosales-Robles. 2002. Tillage effects on microbial biomass and nutrient distribution in soils under rain-fed corn production in central-western Mexico. Soil and Tillage Research 66(2): 143152. Doi:10.1016/S0167-1987(02)00022-3.

Sánchez-Cohen, I., H. Macías-Rodríguez, P. Heilman, G. González-Cervantes, S. F. Mendoza-Moreno, M. A. Inzunza and J. Estrada-Ávalos. J. 2006. Multiobjective planning in the irrigation districts of Mexico. A decision support system application. Ingeniería Hidráulica en México 21(3): 101-111. https://www.revistatyca.org.mx (8 de febrero de 2021).

Sánchez-Cohen, I., J. M. Maass M., U. O. Spring, P. Heilman, J. L. González B., G. Diaz P. and M. Velásquez V. 2008. Integrated water management research in Mexico: Opportunity for North American collaboration. Journal of Soil and Water Conservation 63(6): 212-213 Doi: 10.2489/jswc.63.6.212A. 
Sánchez-Cohen, I., F. Barbosa-Moreno, M. A. Macias-Corral, G. Díaz-Padilla and R. A. Guajardo-Panes 2020. A multidisciplinary perspective to protect the quality of water in natural wetlands. A case study in Oaxaca, Mexico. Journal of Water Sanitation and Hygiene for Development 10(2): 366-373. Doi: 10.2166/washdev.2020.014.

Sifuentes-Ibarra, E., W. Ojeda-Bustamante, R. E. Ontiveros-Capurata and I. Sánchez-Cohen. 2020. Improving the monitoring of corn phenology in large agricultural areas using remote sensing data series. Spanish Journal of Agricultural Research 18 (3): e1204, 13 pages. Doi:10.5424/sjar/2020183-16269.

Sotelo R., E. D., C. Ortiz T. y M. I. Rizo A. 2005. Áreas potenciales para el cultivo de pitahaya (Hylocereus undatus (Haw.) Britt. \& Rose) en el sur del Estado de México. Ciencia Forestal en México 30(98):87-97. https://cienciasforestales.inifap.gob.mx/editorial/index.php/forestales/article/view/850 (8 de febrero de 2021).

Van Eck, N. J. and L. Waltman. 2007. VOS: A New Method for Visualizing Similarities Between Objects. In: Decker, R. and H.-J. Lenz (Eds.). Advances in Data Analysis. Springer. Freie Universität. Berlin, Germany. pp. 299-306. Doi:10.1007/978-3-540-70981-7_34.

Villanueva-Díaz, J. D., W. Stahle, B. H. Luckman, J. Cerano-Paredes, M. D. Therrell, M. K. Cleaveland and E. Cornejo-Oviedo. 2007. Winter-spring precipitation reconstructions from tree rings for northeast Mexico. Climatic Change 83(1): 117-131.

Doi: $10.1007 / \mathrm{s} 10584-006-9144-0$.

Villanueva-Díaz, J., E. A. Rubio-Camacho, Á. A. Chávez-Durán, J. L. Zavala-Aguirre, J. Cerano-Paredes y A. R. Martínez-Sifuentes. 2018. Respuesta climática de Pinus oocarpa Schiede ex Schltdl. en el Bosque La Primavera, Jalisco. Madera y Bosques 24(1): e2411464. Doi:10.21829/myb.2018.2411464. 
Villanueva-Díaz, J., D. W. Stahle, M. D. Therrell, L. Beramendi-Orosco, J. EstradaÁvalos, A. R. Martínez-Sifuentes, C. C. Astudillo-Sánchez, R. Cervantes-Martínez and J. Cerano-Paredes. 2020. The climatic response of baldcypress (Taxodium mucronatum Ten.) in San Luis Potosi, Mexico. Trees - Structure and Function 34(2): 623-635. Doi: 10.1007/s00468-019-01944-0.

Viso, A. M. 2005. Sustainability and governance. Arbor 181(715): 317-331. Doi:10.3989/arbor.2005.i715.415.

Waas, T., L. Hugé, A. Verbruggen and T. Wright. 2011. Sustainable Development: A Bird's Eye View. Sustainability 3(10): 1637-1661. Doi:10.3390/su3101637.

Zamora M., B. P., M. C. Zamora-Martínez, M. C. C. Nieto P. P y F. T. A. García C. 2018. Condiciones edáficas, abundancia y riqueza de hongos ectomicorrízicos comestibles. Revista Mexicana de Ciencias Forestales 9(48):226-251. Doi: 10.29298/rmcf.v8i48.152.

Zarta Á., P. 2018. La sustentabilidad o sostenibilidad: un concepto poderoso para la humanidad. Tabula Rasa (28): 409-423. Doi:10.25058/20112742.n28.18.

\section{(c) (i) (9)}

Todos los textos publicados por la Revista Mexicana de Ciencias Forestales -sin excepción- se distribuyen amparados bajo la licencia Creative Commons 4.0 Atribución-No Comercial (CC BY-NC 4.0 Internacional), que permite a terceros utilizar lo publicado siempre que mencionen la autoría del trabajo y a la primera publicación en esta revista. 